\title{
The Unified Spacememory Network: from Cosmogenesis to Consciousness
}

\begin{abstract}
Nassim Haramein, William David Brown, Amira Val Baker
ABSTRACT

The recent developments of advanced models of unified physics have brought a deeper understanding of the fundamental nature of space, time, energy and matter. It is becoming apparent that information and geometry are primary to explaining these fundamental agents. In previous work, we demonstrated that the subatomic nucleon structure of the proton and recently the electron can be derived directly from a spacetime holographic structure of Planck-scale quantum vacuum oscillators fluctuating as spacetime pixels, demonstrating that spacetime at the very fine level of the Planck-scale is discrete with information quanta. We have found that when considering the granular spacetime information-energy structure from which we demonstrate matter and mass arises, the phenomena of self-organizing systems that leads to self-awareness and consciousness is integral to-and a natural emergent property of the feedback-dynamics of spacetime information itself. In this work, we describe how the integral function of the information feedback dynamics of spacetime, which engender mass-energy, is the missing element in understanding the evolution and development of self-organizing physical systems in general, and the emergence of the biological organism in particular. We evaluate non-classical quantum mechanical phenomena of physical and biological systems in light of the Maldacena-Susskind holographic correspondence theorem from which an equivalence of wormhole spacetime geometry and quantum entanglement is derived. We suggest that the Planck-scale micro-wormhole entanglement structure of multiple spacetime coordinates engender the macromolecular assemblies of living cells, and that this wormhole-entanglement may function in the memory and learning capacity of the biological entity. Furthermore, the recursive information encoding feedback processes of the quantum spacetime micro-wormhole network, which we refer to as spacememory, enables memory and learning in physical systems across all scales, resulting in universal evolutionary tendencies towards higher levels of ordering and complexity - foundational to evolution, sentience, and awareness.
\end{abstract}

Key Words: quantum spacetime, consciousness, micro-wormhole, entanglement, macromolecules, Planck scale, fractal, holographic, cosmology, biogenesis DOI Number: $10.14704 / \mathrm{nq} .2016 .14 .4 .961$

NeuroQuantology 2016; 4:

\section{Introduction}

In the $20^{\text {th }}$ century, models of relativity and quantum theory gave the observer a central position within the physical processes of the Universe. The purely mechanical idea of matter and energy interacting like clockwork under the auspices of the unmalleable laws of physics have since evolved into the concept of the dynamical interactivity of systems, represented not only as an exchange of matter and energy, but as well, of information. Not surprisingly, information is

\section{Corresponding author: William Brown}

Address: TorusTech LLC, 991 Calle Negocio, San Clemente, Ca 92673

Phone: +1 928-607-9569

e-mail $₫$ william@torustechllc.com

Relevant conflicts of interest/financial disclosures: The authors declare that the research was conducted in the absence of any commercial or financial relationships that could be construed as a potential conflict of interest. 
central to many provisional explanations of consciousness (Tononi, 2008). This has led some researchers to take a closer examination of the relationship between consciousness and reality (Penrose et al., 2011).

Consciousness has been a controversial subject within science, as it is not just about explaining a particularly complex phenomenological state of the brain - it pierces right to the heart of our conception of the material world. An investigation of the nature of consciousness, as it turns out, is inextricably linked with the exploration of the nature of reality. This is epitomized in the centuries-old adage "if a tree falls in the forest, and no one is around to hear it, does it make a sound?" To what degree and extent is objective reality dependent on the observer? Clearly, most of us would agree that of course it makes a sound, as sound is just mechanical vibrations propagating in air molecules.

Yet, this question has resurfaced in the form of Schrödinger's cat, posed in part to demonstrate the non-physical nature of the Heisenberg-Bohr model of quantum theory, also known as the Copenhagen Interpretation, which is the predominant quantum mechanical model. Such models arose from attempts to interpret the physical mechanisms of the famous double slit experiment, which were considered by some physicists to have no classical explanation. However recent experimental studies find a different interpretation of the double slit experiment, based on pilot waves in fluid dynamics of classical systems (Bush, 2015; Couder and Fort, 2006; Borghesi et al., 2014). The Copenhagen interpretation has led to the typical inference that the observer and the observed can be isolated from the system they are embedded in; i.e. all other frames - in that their relationship defines the reduction of the probability amplitude (collapse of the wave function) into a definite event. In such a model, the wave function $\psi(x, t)$ that describe the superposition of eigenvalues is taken to translate as a probability amplitude, and a particle has no real physical existence until it is somehow observed.

The concept that an observer generates the reality in which the event occurs, such as sound emission by the falling tree, assumes an isolation of the frame of reference relative to the event. That is, all interactions in the system, the air molecules for instance, the birds in the neighboring tree, the microbial life all around and in the tree and so on, can all be considered subsystem frames "observers" - a point recently expounded by others as well (Susskind, 2016). experiencing the event from different perspectives. Is there a mechanism in which the relationship of reference frames generates a collective behavior that eventually evolves to a self-awareness state?

Even from an evolutionary perspective, if consciousness is simply an epiphenomenological accident of brain neurology, the question still remains, why did consciousness evolve at all? Many researchers have demonstrated that pure algorithmic computational functions are completely sufficient to determine everything from the complex behavior of flocking birds and schools of fish, to simple behaviors of avoiding prey, finding food, and mating. As such, wouldn't the evolutionary development of consciousness, and certainly self-awareness, be superfluous? Following the consensus model of evolution and development, consciousness would not arise, and indeed this is what many theorists have posited (Dennet, 1991; Dawkins, 1989). Essentially asserting that consciousness is just an illusion, wherein the illusory experience of what we call consciousness occurs after all mechanical and computationally determined processes have occurred, suggesting that we are no more than genetically preprogrammed automatons, as are all other organisms.

Such inferences result from considering systems reduced to their component subunits, and thus largely fail to consider the emergent properties of systems which have complex interactions at different scales. Certainly at the subatomic scale, systems may have non-linear and nonlocal interactions that are beyond what a linear summation of the parts would predict. When such nonlocal quantum phenomena are extended to the macromolecular environment of the biological system, unpredictable systemsbehavior may emerge, and may be a critical aspect of information, awareness, and sentience processes of living matter. In this context and for the purpose of defining the local and nonlocal interactions as a collective behavior as well as generalizations of entanglement (Maldacena \& Susskind, 2013), the holographic principle (Haramein, 2013), and emergent properties, we thus utilize the terminology synergetic (introduced in Fuller \& Applewhite, 1982). From considerations such as these, it may be that awareness is a non-local mechanism arising from, and intrinsic to the interactivity of Planck-scale 
spacetime geometry that enables nonlocal and nonlinear signal integration (we refer to this multiply-connected spacetime geometry as the micro-wormhole network), forming an integral function within physical and biological processes. The information dynamics underlying physical processes would therefore require a selforganizing framework emerging from characteristics such as intercommunicativity, memory, hysteresis, iterative feedback mechanisms, retrocausal influences, and local and nonlocal interactions, which we refer to as spacememory.



Figure 1. Potential paths of the evolution of matter in the Universe (for conceptual illustration only). Arrows indicate the relative degree of probability under conventional models, with potential path 1 having the strongest degree of probability, but the lowest degree of order and complexity; potential path 2 having the lowest degree of probability, but the highest degree of ordering and complexity; and potential path 3 having a median probabilistic expectation value.

As such, we describe how underlying nonlocal information dynamics, intrinsic to the properties and behavior of material systems and uniquely harnessed by the natural nanotechnology of supramolecular systems of the brain (similar to the Hameroff-Penrose model of orchestrated objective reduction (Hameroff \& Penrose, 2014)) are involved in producing the sentience, awareness, and memory of cognitive processes. Moreover, we propose that nonlocal influences across spatial and temporal domains, communicated through the micro-wormhole network of the Planck-scale geometric structure of spacetime, may play an instrumental role in the evolution and development of physical systems, thus engendering an ordering dynamic as well as directionality towards higher levels of complexity and organizational synergy. This ordering dynamic is the same, from the evolution and development of physical systems to biological systems, and in fact, may serve as a defining characteristic of life itself. In this sense, the conception of a living system extends beyond the normal consideration of only the biological organism (e.g. evolution and synergism are not merely products of the biosphere, but are present in the interactivity of systems in general leading to the emergence of the biosphere) and properties of sentience and awareness are involved in the directed (non-random) generic evolution of matter and the universe.

\section{Evolution and Development Under the Stochastic Model of Modern Cosmology}

Considering the cosmogenic evolution of matter, there are several possible evolutionary paths that we can imagine occurring. Figure 1 gives a hypothetical example of 3 potential paths, and their relative probabilities under a purely stochastic model.

In potential path 1 the initial conditions such as a high or low value for the fine-structure constant, prevents nuclear binding which ultimately results in a diffuse homogenous proton-electron plasma. Complexity is not achieved. In potential path 3 , the conditions are favorable to nuclear binding allowing polyatomic molecules to form. However, conditions promoting biogenesis are not present and only lifeless molecular aggregations form. Minimal complexity is achieved. Now consider potential path 2, not only do polyatomic molecules form, but conditions (as observed in our present universe) are such that they are ordered into complex higher molecular conformations, perhaps producing a rudimentary metabolism (Braakman \& Smith, 2013) and structures that are able to self-catalyze and therefore replicate (Monnard \& Ziock, 2008; Attwater et al., 2010; Dworkin et al., 2000; see also Figure 2). Through deviations/variations of the replication process and natural selection of functionality, these differentiate into diverse forms, which is to say evolve. Complexity is achieved.

The evolutionary pathway of the DNAmRNA-tRNA/rRNA transcription and translation system, which is the information system at the heart of all biology, emphasizes the gap in the description between the observed extant 
complexity and the putative processes that would have fostered, or even driven, the emergence of such complexity. Consider again the ribozyme (Figure 3). The ribozyme (image 3A) represents the possible link in the chemical evolution between information encoding molecules and catalytic enzymes. Small ribozymes would have been the primordial precursors of the ribosomal RNA (image 3B), the entire complex, or holoenzyme, of which is perhaps one of the most complex molecular structures that we know of, and is one of the three fundamental pillars of the molecular biological processes underlying all living organisms (the other two being the DNA molecule, and the DNA/RNA polymerase machinery). There are over 100 naturally occurring nucleotides (generated by modifying the 4 canonical ribonucleosides) that make up the rRNA molecule (Cantara et al., 2011). If each position of the rRNA subunits were to be tested with each of the 100 possible nucleosides, then with a length of 4448 nucleotides in some species (Brosius et al., 1978 \& 1980), there are $100^{4448}$ different possible configurations - that is $1.0 \times 10^{8896}$ possible first order configurations. mechanism of feedback interaction between universal structures than the one given by Fred Hoyle (Hoyle, 1983). Hoyle calculated the probabilities of a blind person ordering the scrambled faces of a Rubik cube. The calculations demonstrated that, due to the fact that the blind person does not know if he or she is getting closer or further to the objective on each move, the probabilities of matching the six colors on each face of the cube are on the order of 1:1 to $1: 5 \times 10^{18}$. Thus, if that person was to labor at a rate of one move per second, it would take $5 \times 10^{18}$ seconds to complete all possibilities. That is to say that it will take up to 158 billion years for that person to reach the goal. Clearly that time period not only grossly exceeds the life expectancy of the Rubik cube player, but it exceeds the lifetime of the Earth or for that matter the existence of our Universe since its estimated inception some 13.7 billion years ago. However, if the blind person is given a simple piece of information, something like a "yes" or "no" prompt every time a move is made, which is every second, then the time needed to complete the Rubik cube equation is drastically reduced to two minutes.

However, no example gives a better mathematical perspective on the necessity for a
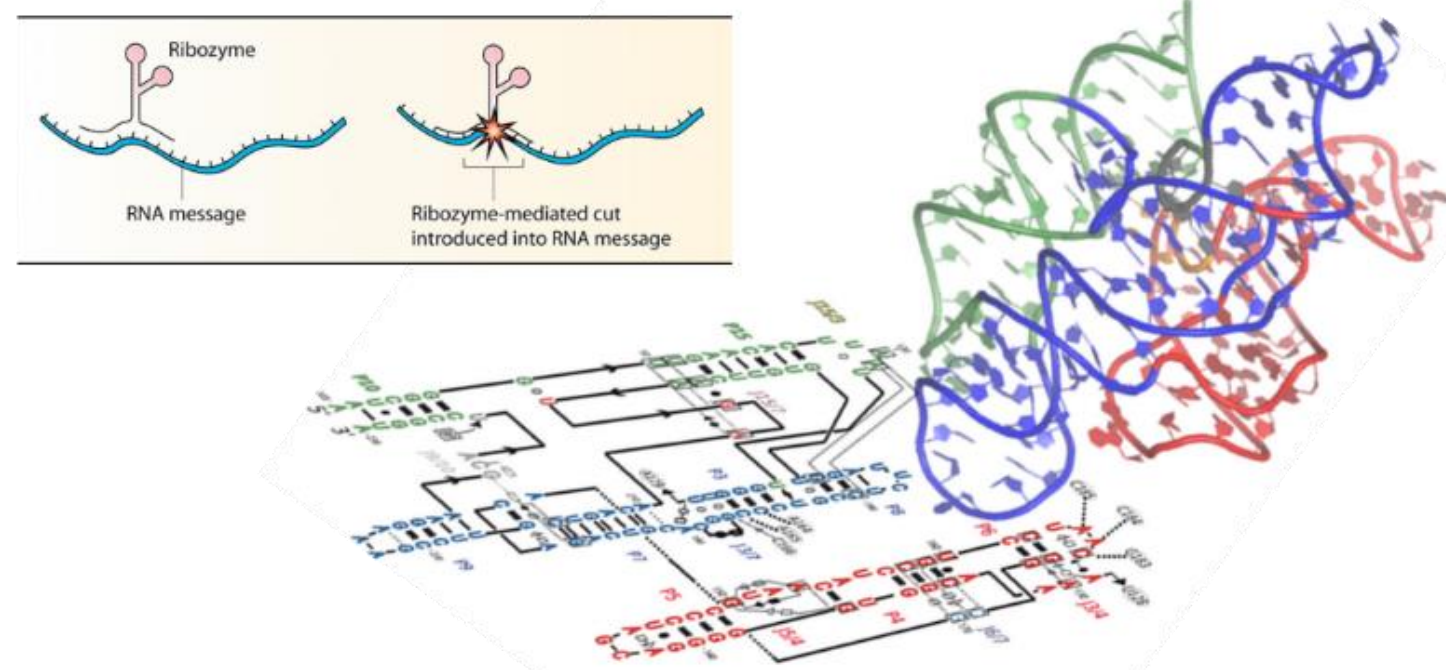

Figure 2. The ribozyme: a ribonucleic acid molecule that is able to perform catalyzing and self-catalyzing enzymatic reactions. It represents a potential informational structure and processor that is the prebiotic progenitor to the DNA-mRNA-tRNA/rRNA transcription and translation multimeric, macromolecular complex of cellular systems. As such, it may represent an evolutionary "link" in the development of information structures. The potential for such structures to arise in the eutectic metal ion ice-water interfaces of pre-cometary bodies opens the possibility that informational structures may arise in specific environments of interstellar space, as a natural evolution of matter. As such, the moleuclar informational structures of prebiotic life may be ubiquitous, and simply one of a number of iterative steps in the development of matter from the fundamental information processing dynamics of the universal spacetime manifold. Information structures are not the exception; they are the rule. 
A

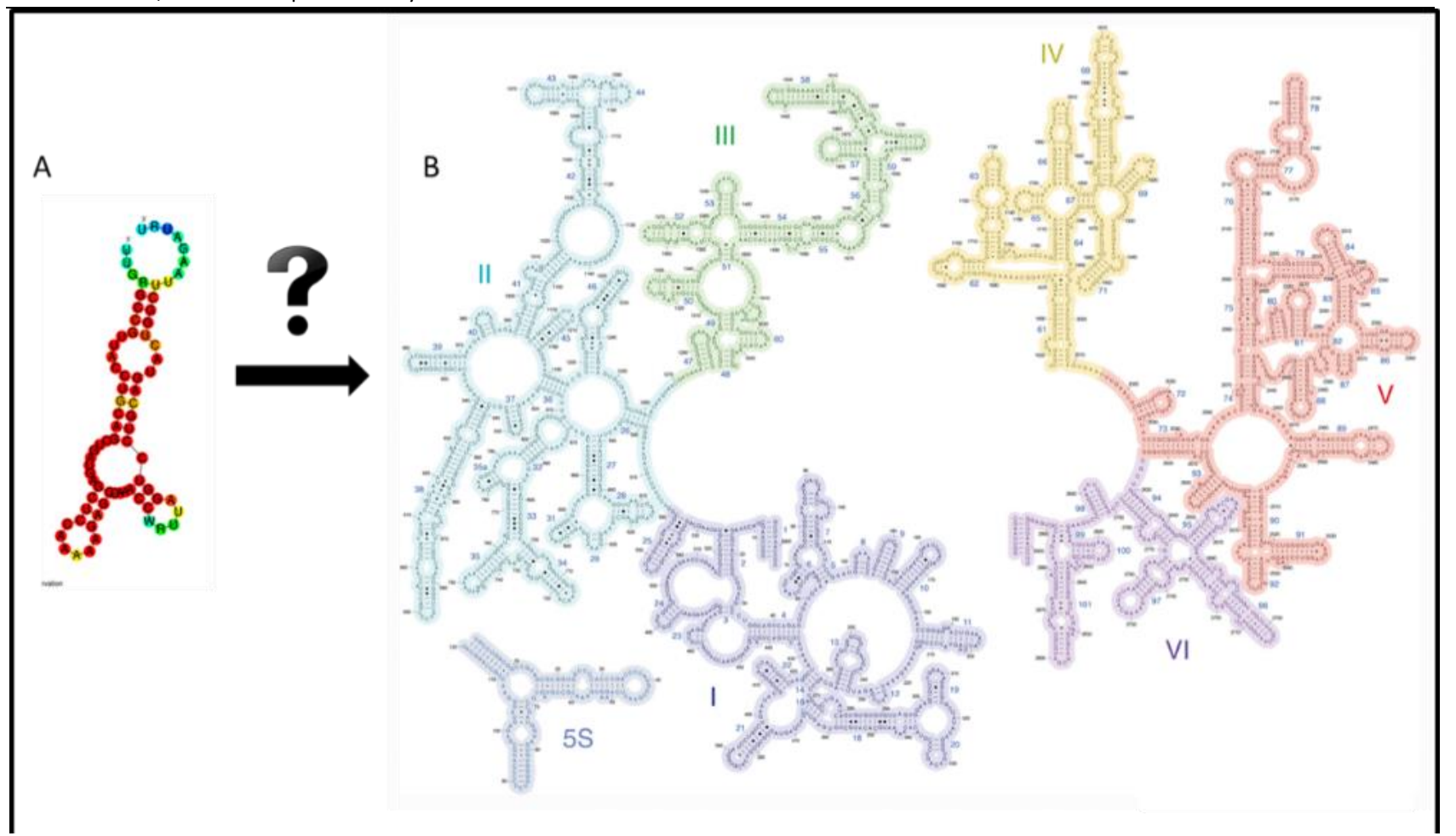

B

III
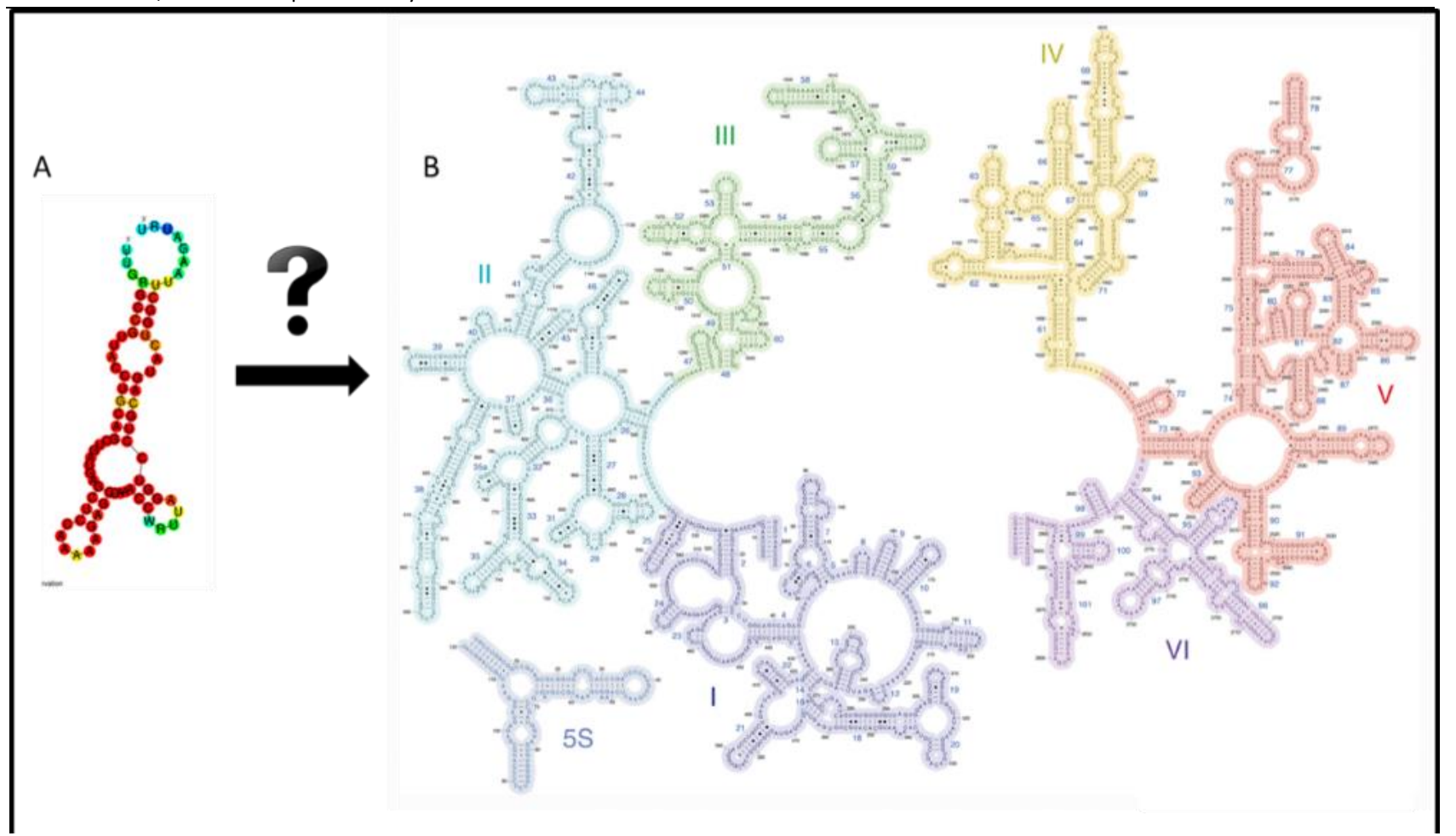

Figure 3. The comparison of simple ribozyme with the complex multi-domain ribosomal RNA. (A) An RNA molecule capable of enzymatic action (Ribozyme), such catalyzing molecules ostensibly serving as the primordial precursors before the transition to the DNA-mRNA-rRNA transcription and translation system. (B) The RNA ribosomal subunits of Escherichia coli, depicting higher orders of structural complexity and organizational synergy over that observed in (A).

The hyperastronomical number of possible configurations to evolve from Figure $3 \mathrm{~A}$ to Figure 3B demonstrates the rationale behind positing that there could be an underlying mechanism producing purposeful innovations, and attracting towards higher levels of structural ordering and functionality. As Andreas Wagner states it: natural selection can preserve innovations, but it cannot create them... nature's many innovations, some uncannily perfect, call for natural principles that accelerate life's ability to innovate (Wagner, 2014). The universe simply hasn't been around long enough for random mutations to test even a minute fraction of the possible configurations of the rRNA molecule, not to mention other biomolecules such as proteins and $2^{\text {nd }}$ or $3^{\text {rd }}$ order configurations involved in protein or RNA folding.

Following the 'weak anthropic principle' (Barrow \& Tipler, 1986), while a state of meaningful ordering, structural, and organizational complexity as that exhibited by living systems is so exceedingly improbable to happen by pure random chance that it is nearly negligible - in a practically infinite set of universal creation events (the multiverse) it will nevertheless occur. This form of speculative hypothesis does not appear to make any falsifiable predictions (Smolin, 2004). However, there are other models (Smolin, 2010; Vidal, 2010) that are not only massively more explanatory, they make predictions, are testable, and as with our particular model: provide a casual mechanism and may unify various physical properties of the Universe such as forces, constants, laws, and perhaps, biological systems and consciousness.

\section{The Planckian micro-wormhole information network}

In previous work, Quantum Gravity and the Holographic Mass (QGHM) (Haramein, 2013), a quantized solution to gravity was given in terms of Planck Spherical Units (PSUs) in a generalized holographic approach. In recent work, the solution has been extended to the electron cloud of all elements of the periodic table with extreme precision (Haramein and Val Baker, 2016). We consider the structure of spacetime at this scale, as not only is it pertinent to understanding Planckian gravitational mechanisms, but also, as we will show, the potential information content and associated communicativity conduits that may enable nonlocal interaction across spatial and 
temporal domains. This spacememory structure serves as the template, integration, and processing substratum for evolution and development of physical and biological systems. In quantum gravitational approaches, space at the Planck scale is quantized, comprised of discrete units that we refer to as PSUs, which serve as informational bits (Planck voxels), where one PSU volume ( $\left.V_{l s}\right)$ is:

$v_{\ell s}=\frac{4}{3} \pi \ell_{r}^{3}=2.21 \times$ $10^{-99} \mathrm{~cm}^{3}$

(where the Planck radius, $\ell_{r}=\frac{\ell}{2}$, and $\ell$ is the Planck length).

The Planck density or the quantum vacuum energy density $\rho$ was subsequently more appropriately calculated in terms of PSUs:

$$
\rho=\frac{m_{\ell}}{V_{\ell s}}=9.86 \times 10^{93} \mathrm{~g}
$$

(where $m_{\ell}$ is the Planck mass). The mass-energy density of any spherical body can therefore be considered in terms of its PSU packing, and can be defined as a fundamental ratio, $R$, of any spherical volume, $V$, to PSU,

$R=\frac{V}{V_{\ell s}}$,

where the corresponding mass-energy, $R_{\rho}$ can thus be calculated in terms of Planck mass:

$R_{\rho}$

$=R m_{\ell}$

In the case of the proton, the Planck vacuum energy density is calculated as,

$R_{p}=R m_{l}=2.46 \times$

$10^{55} \mathrm{~g}$,

which is equivalent to the mass of the Hubble volume (observable universe),

$N_{\text {edd }} m_{p}$

$=2.62602 \times 10^{55} \mathrm{~g}$,

(where $N_{\text {edd }}$ is the Eddington number and is = $1.57 \times 10^{79}$ protons and $m_{p}$ is the proton rest mass (Mohr, Taylor, \& Newell, 2012)), indicating that the mass-energy of all the protons in the Hubble volume may be stored in the virtual mass-energy density of any one proton.

Following the holographic principle (Hooft, 2001), based on the Bekenstein-Hawking formulae for the entropy of a black hole (Bekenstein, 1973; Hawking, 1975), Haramein defines the holographic bit of information as the equatorial disc of the oscillating PSU. These PSUs, tile over the area of the proton surface horizon, producing a holographic relationship with the interior information vacuum mass-energy density. The ratio of the information bit to the spherical surface is thus defined as

${ }^{\eta}=\frac{A_{s}}{A_{\ell c}}$

(where $A_{s}$ is the surface area of the spherical body and $A_{\ell c}$ is the area of the PSU equatorial disc).

It is then shown that the ratio of this interior vacuum energy density to the surface horizon Planck tiling yields an exact quantized derivation of the Schwarzschild solution to Einstein's field equations, and thus a quantized approach to gravity is found, where

$m_{h}=\frac{R}{\eta} m_{\ell}=\frac{r c^{2}}{2 G}=$

$m_{s}$

(where $m_{s}$ is the Schwarzschild mass of the black hole). Clearly a surface-to-volume ratio of the Planck vacuum information energy is equivalent to the mass-energy of the system and therefore generates a quantized information network perspective, akin to the biological system where the cellular membrane is the primary information interface.

The solution is then applied at the nucleon scale, where the mass of the proton is found to be:

$$
\begin{aligned}
m_{p}=2 \frac{\eta}{R} m_{\ell} & =2 \phi m_{\ell} \\
& =1.673342 \\
& \times 10^{-24} \mathrm{~g}
\end{aligned}
$$

(where $m_{p}$ is the rest mass of the proton and $\phi$ is the fundamental universal ratio defined as $\frac{\eta}{R}=$ $3.84 \times 10^{-20}$ ). The radius of the system can then be extracted by this simple algebraic relationship,

$$
=4 l \frac{m_{l}}{m_{p}},
$$

(where, $r_{p}$ is the radius of the proton computed from Planck discrete quantities. The resulting radius is within one standard deviation of the latest muonic measurement of the proton (Pohl, 2013), and therefore the most accurate charge radius measurement prediction from theoretical tenets to date. 
As described in QGHM, we suggest quantum vacuum oscillators assume a coherent structural organization of spacetime to form the proton, and the entire information density of the cosmological volume defined by the Hubble volume (which obeys the Schwarzschild condition) is holographically encoded within the proton. The value for $\eta$ is calculated for the proton where it was shown that there are $\sim 4.33 \times 10^{40}$ Planck voxels tiled over the surface horizon of the proton.

In this geometric model, and following the Schwarzschild solution,

$d s^{2}=-\left(1-\frac{2 m}{r}\right) d t^{2}+\left(1-\frac{2 m}{r}\right)^{-1} d r^{2}+r^{2}\left(d \theta^{2}+\right.$ $\left.\sin ^{2} \theta d \varphi^{2}\right)$,

we note that the oscillating PSU satisfies the Schwarzschild solution of an Einstein-Rosen bridge,

$l_{r} \ll \frac{2 G m_{l}}{c^{2}}$,

As such, following from the calculations above, we can conclude that there are $\sim 4.33 \times 10^{40}(\eta)$ wormhole terminations over the area of the surface horizon of the proton.

Note from equation (11), when $r<2 m$, then $r$ becomes a timelike coordinate and $t$ becomes spacelike, and the interior of the event horizon is non-static. This becomes pertinent in the consideration of superluminal, or quasiinstantaneous interactions through the microwormhole network, as signals passing through the wormhole interior are not so much traveling through 'space', but through a time-like coordinate system.

While static-wormhole solutions appear unstable, models with spin and charge, such as Kerr-like wormholes (Miranda et al., 2013), have been calculated to be stable solutions. And furthermore link well with the expansion factor of spacetime generally referred to as 'dark energy', in that there may very well be a pervasive and ubiquitous field of negative energy that could support a Planckian wormhole spacetime architecture (keep in mind for instance that within the standard model the electron has infinite negative mass before vacuum renormalization). Furthermore, in an effect related to the cosmological constant (Weinberg, 1989), it has been empirically demonstrated by Casimir dynamics (Nation et al., 2010) that the weak energy condition (the axiom that any physical system must have a positive energy density) is eISSN 1303-5150 violable (Lemos et al., 2003). This implies that it is possible for a wormhole to be sustained, that is to say traversable. It is important to note that even in classically defined unstable wormholes, the collapse of the throat is restricted to the Planck length in diameter, such that the micro-wormhole network, comprised of wormholes of the Planck length in diameter, will be highly stable (existing since the big bang).

If no less than two Planckian surface horizon wormholes connect to two other protons, then following the connection network would result in a geometric increase in the number of connected protons, soon including all $\sim 10^{80}$ protons of the observable universe. Since hadrons form the nucleus of all atomic matter, it implies that not only is spacememory built from the quantum entangled wormhole network, but matter is as well. Given that there are $\sim 10^{40}$ Planckian wormhole terminations along the surface horizon, there are myriad spatial and temporal connectivity configurations available to any single proton.

\section{The Correspondence of Quantum Entanglement with the Planckian Wormhole Architecture of Spacememory}

If we consider a spacememory architecture of multiply connected protons, how would this potential quantum communication network influence the interaction of matter, especially matter within the biological system? For instance, might the brain-body system be connected through a spacememory micro-wormhole network via the unique structural, electromagnetic, and quantum resonant characteristics of supramacromolecular assemblies of which it is comprised? Recent work by Raamsdonk, Maldacena, and Susskind equate the spacetime structure of wormholes (ER bridges) with the phenomenon of quantum entanglement (EPR correlations), so that ER = EPR (Maldacena \& Susskind, 2013; Raamsdonk, 2010; Baez \& Vicary, 2014). Both of these phenomena, the former from Relativity and the latter from quantum theory, involve nonlocal, i.e. apparent superluminal (quasi-instantaneous) signal transmission.

Several notable theories have been expatiated involving non-classical quantum effects in living matter. Although the role of quantum effects in the biological system have only 
recently moved from theoretical to empirical investigations, it is highly probable that biological systems have a robust utilization of what is termed non-classical, or quantum behavior. We note as well that it is reasonable to consider such explanatory models given that there have been many billions of years to select for such capabilities, by a system that produces observably remarkable capabilities, and that biological systems fundamentally utilize biomolecular nanomachines to manipulate subatomic and atomic matter, which falls squarely in the quantum domain (the size at which quantum effects are shown to be physically relevant). Theoretical work initially began with investigating the potential of long-range coherence and energy efficiency in metabolic processes -- specifically Fröhlich-Bose-Einstein condensates (Fröhlich, 1968) -- and eventually extended to considerations of possible quantum effects underlying neurological dynamics and consciousness (Freeman, 2006).

Of particular credence is the proposed quantum phenomena involving microtubules, as described in the Penrose-Hameroff model of Orchestrated Objective Reduction (Hameroff \& Penrose, 2014), which posits a quantum gravitational mechanism of state reduction, each event of which represents a proto-conscious information processing event of spacetime. Through massive parallelism and quantum coherency this mechanism is employed in an orchestrated fashion within the supramolecular information processing structures of the cell, specifically the cytoskeletal microtubule network. Empirical work has observationally verified nonclassical quantum mechanical phenomena within the biological system (Hildner et al., 2013; Panitcharyangkoon et al., 2010; Hayashi and Stuchebrukhov, 2010). Other research has empirically demonstrated the memory capabilities, signal conductivity, nested fractal resonances and singular state mechanics of microtubules (Bandyopadhyay, 2013; Sahu et al., 2013). Continuing this line of inquiry, we posit that the supramacromolecular microtubule/mitochondrial reticular matrix (Thar \& Kuhl, 2004) may be involved in the processing of information at the quantum level, and that nonlocal and retrocausal signals are strong influences on the non-random behavior of key macromolecules, such as DNA.

Microtubules are a key structural filament of the cytoskeletal network, others being actin and eISSN 1303-5150 intermediary filaments. Microtubules are biopolymers formed by the covalent binding of protein heterodimers referred to as $\alpha, \beta$, and $\gamma$ tubulin. When a GTP molecule is transferred to a tubulin heterodimer it polymerizes with other GTP-tubulin residues to form a protofilament; typically, 13 protofilaments will arrange to form a long cylindrical tube with an inner luminal space occupied with atomically ordered water, ions, and in some cell types luminal particles are observed (Garvalov et al, 2006). In addition to luminal particles, there are a number of regulatory proteins that bind to the outer surface of the microtubule, such as tau proteins, motor proteins like dynein and kinesin, and other microtubule associated proteins (MAPs). As in the inner lumen, charged ions are conducted along the outer surface such that microtubules have a nonlinear conduction of electrical current. In this work, we will examine aspects of how tubulin residues of microtubulin polymers can share and integrate information across large domains of the brain and body by interaction through the micro-wormhole network of spacememory. Moreover, we will further examine how the Planckian wormhole network of the microtubule/mitochondrial reticular matrix may intercommunicate information with spatial and temporal frames that are far distant from the body, enabling signal nonlocality.

The multiple connections of the brainbody spacememory micro-wormhole network may engender memory, nonlocal interaction and massively parallel, quasi-instantaneous information processing. Whereas conventionally the outcome of quantum critical events is thought to be purely stochastic, the spacememory network allows for the nonlocal transmittance of influences from spatial and temporal domains that would normally be isolated by the speed of light restriction to signal transmission. Much like the so called superposition of quantum mechanics, this means that the mind, like the quantum mechanical particle that becomes extended in space and time, is not necessarily localized to a definite position (or timeframe). As such, there are sensory data available to the brain-body system that extend much beyond the normally defined inputs (Sheldrake, Scientific papers on telepathy, the sense of being stared at, and Morphic Resonance, 2014; Hameroff, How quantum biology can rescue conscious free will, 2012). It is important to note that the connectivity does not necessarily need to be entanglement, such as that described in a pure 
quantum state, more so the weak coherence that can occur in mixed states, such as that described by quantum discord (Ollivier \& Zurek, 2001).

Considering this, the macromolecular and cytoarchitectonic structures of the brain are not so much involved in producing a virtual re-creation of the "external" environment, but receiving the information and recording it, as well as orchestrating responses. In this sense, the brainbody system operates much more as a receiver and antenna with reply-back operations, recording the information in spatial configurations of myriad neuronal synaptic, subsynaptic, and supramacromolecular assemblies. However, this type of behavior may not be restricted only to the brain, but more so that the brain arises from such behavior of physical systems in general, albeit as a highly refined and paramount example of such intrinsic spacetime proto-conscious phenomena, and as such at this more basal level there is a continuum between the "external" world and the "internal" world, a principle of ubiquity.

A significant challenge facing consciousness science, and therefore existentially science itself since all data are ultimately interpreted by human consciousness, is the supposed dichotomy between an objective external reality and the internal subjective experience of the conscious entity. If all experiences are merely virtual re-creations within the synaptic networks of the brain, then we only ever experience a simulacrum of the external environment, never actually experiencing reality as it objectively exists. The unified spacememory network offers an alternative explanation for the phenomenology of experience by the conscious entity as well as the supposed objective/subjective dichotomy of conscious experience. The information processing events taking place within the synaptic and subcellular networks of the organism are not only strongly correlated within, but entangled with the external environment as well.

This means that quantum critical events within the subcellular macromolecular environments of the organism are connected with "external" events to a much higher degree than would classically be anticipated. Most salient of all, is that "external" events -- the supposed objective reality -- are influenced by the internal processes of awareness occurring within the organism. To put it another way, the universe "knows" subjective qualia, such as the color blue, through our experience of the color blue, and in this way putatively subjective qualia have an external objective reality. Recall that within conventional consciousness science the color blue (and any other subjective qualia) is only ever experienced in the virtual re-creation of the world by the brain. Objectively there would be no such thing as the color blue, aside from a particular wavelength of electromagnetic radiation that the brain interprets as "blue", and as such "blue" is only a symbol within the simulacrum of reality that is experienced through the machinations of the mind. The prediction that emerges from this hypothesis is that supposedly subjective qualia will have a meaningful and measurable influence on external objective events, and are therefore not entirely subjective at all.

\section{The Role of Ordered Water in Coherence and Information Transmission Within the Biological System}

Electromagnetic phenomena and the physicochemical properties of atomically ordered water are elementary to the functionality of the biological system, as all living systems ultimately emerged from a medium of water. Yet even given the central and fundamental importance of such phenomena they have been disproportionately overlooked or altogether ignored within conventional considerations and investigations of the cellular and subcellular environment of the organism. This is in no small part due to a profound misunderstanding of the nature of the biological organism, in which it is often times far from being appropriately recognized as a naturalengineering marvel of superlative nanotechnology, the likes of which we are nowhere close to being able to replicate. A system that can manipulate and order matter on the subatomic and atomic scales with supreme precision and sophistication may very well have some capabilities that are far more clever than the limited propensities that have classically been considered as operating within the domain of the biological organism.

In examining ordering parameters of the spacememory field, and the resulting non-random behavior of physical systems that has been hypothesized, we identify effects at the quantum scale and associated non-classical phenomena as an important intermediary between macroscopic order and the influences of spacememory. It has 
naively been presumed that non-classical behavior of quantum mechanical phenomena would be largely absent or strongly stifled within the biological organism, in part because it is a macroscopic, many-bodied system as well as because it operates at much higher temperatures than those at which quantum effects had historically been observed in laboratory experiments. However, many-bodied entanglement of macroscopic objects has now been observed at room temperature (Lee et al., 2011). Such results have not properly been taken into account, as well as other key factors within the biological organism that may mitigate events leading to decoherence, such as the atomically ordered water of the subcellular and interfacial environment and coherence with the spacememory field of the quantum vacuum.

Microtubules were one of the first macromolecular structures to be suggested to involve non-classical quantum mechanical phenomena in information processing, in part because the tubulin dimers and protofilaments form luminal spaces that may be sufficiently shielded from the thermal noise of the cellular environment that would lead to rapid decoherence. Additionally, and of key importance, is the role of water in functioning as the intermediary between collective, organized behavior and ordering parameters of biomolecules and the spacememory field. This is in part due to the enabling of the quantum coherence and information processing that may be occurring by thermally isolating certain subcellular domains for physiologically relevant times (as long as a 100 milliseconds or more). Such as in the microtubules where ordered water layers of atomic dimensions effectively form quantum electrodynamic resonance cavities (Mavromatos et al., 2002) which can maintain coherent and entangled electronic modes for relatively long periods of time.

Water is instrumental in defining the 3dimensional conformation of protein folding from the linear peptide chain that emerges during translation by the ribosome (Zhang et al., 2009), as well as mediating the specific interaction of proteins with their respective substrates, such as in DNA-polymerase binding of specific promoter loci for gene transcription (Fuxreiter et al., 2005; Yang et al., 2014). As such, there is virtually no cellular or biomolecular function that is not mediated by water. Far from the classical paradigm - water is not an inert theater in which eISSN 1303-5150 the machinations of biochemistry unfold, instead it is much more so the director (Del Giudice et al., 2010).

Electric and magnetic fields, originating either from the electrochemical activity of the cell, coherent electromagnetic transmission originating from mitochondria or internally by energetic pumping (optical lasing of mitochondrial and ordered water origin) will cause the electric dipole moment of water molecules within and around the microtubule to align and oscillate in collective coherent modes. Delocalized electrons of the $\pi$ - orbitals in aromatic amino acid residues of the tubulin dimers of the microtubule will couple with the dipole oscillations of the ordered water of the luminal space, resulting in a quantum coherent solitonic state extending over the entire microtubule, and perhaps the collective network of which it is a part. The QED-cavity nature of the ordered water layers allows for shielding of electrostatic interaction from thermal decoherence, such that quantum coherence can be maintained for physiologically relevant time spans of several 100 milliseconds or more.

The electric dipoles of the water molecules are coupled to the quantum electromagnetic field, which we have shown at the Planck-scale is of such high energy (the Planck energy for a single Plancklength spherical oscillator) that spacetime is in fact highly curved. The curvature is so great that spacetime actually becomes a multiply-connected geometry at the Planckian scale, what the preeminent physicist John Archibald Wheeler (who coined the terms black hole and popularized the term wormhole) referred to as quantum spacetime foam. We have posited and will examine in greater detail in this manuscript how information is intimated nonlocally through the Planckian spacetime micro-wormhole network. In this way, coupling of the dipole moments of water molecules to the quantized electromagnetic field will allow for the emission of photons carrying the information of the spacememory field. The coherent electromagnetic emissions will in turn modulate the electronic behavior of key biomolecules of the cell, which ultimately determines the physicochemical behavior of the system.

Therefore, underlying the matter which comprises living systems, there may be memory imprinting into the Planckian wormholespacememory structure, in which time is a 
function of information on the structure of space during the universal evolution (spacetime = spacememory, i.e. no memory = no time). Memory, and recursive information feedback/feed-forward processes of the quantum vacuum, allow for learning and evolutionary behavior. This applies not only to the mesoscale of the biological organism, but to physical systems ranging from the Planckian to the cosmological scale, and the Universe as a whole. As such, the process of cosmogenesis can be equated with a living, or biological process of iterative evolutionary development - biological cosmogenesis. In this sense, there are living processes occurring at all scales of the Universe, and with memory and learning being functions of awareness - life and consciousness become intrinsic ubiquitous characteristics, embedded in the very dynamics and mechanics of physical processes of spacetime and thus, the Universe itself.

To summarize, one of the most salient structures of supramacromolecular assemblies are the aromatic rings of amino acids and sugars. When specifically, and uniquely structured into large assemblies, the $\pi$ orbitals contain delocalized electrons. Oscillations of the $\pi$ orbitals can be strongly correlated across supramacromolecular assemblies (Wang \& Kais, 2007; Rieper et al., 2011), resulting in strong coherence, and/or entanglement. One of the key aspects that we emphasize is that quantum entanglement may be a function of the Planckian wormhole structure of spacememory. As such, entanglement of specific biomolecular entities is concordant with connectivity circuits of the Planckian wormhole network of the spacememory field. In this way, the cellular and molecular systems of the biological organism are extended through space, time, and the information exchange of the universal Planckian wormhole network holographic imprint on the structure of space, defining the spacememory of the evolutionary process.

We point out the demonstration of quantum phenomena in the meso-scopic domain of biology for the reason that there is no consensus explanation for the mechanisms underlying quantum effects, such as entanglement. However, the fluid dynamic, geometrical, and information properties of spacememory itself have begun to emerge as a possible explanation for the underlying quantum phenomena, even producing demonstrations of such putatively non-classical behavior at the macroscopic scale, see for instance eISSN 1303-5150
Pilot-Wave hydrodynamics (Bush, 2014). As such, this serves as a demonstrable link to the potential role of Planckian spacememory in the biological system in particular and physical systems in general.

\section{Ordering Dynamics of the Universal Spacememory Field}

As we have shown in QGHM, protons emerge from a coherent structural-geometry of Planckian vacuum fluctuations of spacememory. The massenergy gradients and holographic relationships (information structure) of which produce the specific and typical characteristics of atomic matter such as the mass, frequency, and strong binding interaction. Following from equations (11) and (12), which describe the quantum geometry of spacememory as a Planckian wormhole network, we extend this model to the cosmogenic evolution of matter. From baryogenesis of subatomic particles to the solid state properties of large complex molecular assemblies, we suggest that this Planckian wormhole information architecture of spacememory is a system that intrinsically contains a basal-level consciousness and produces an ordering dynamic and directionality to cosmological evolution.

Returning to Figure 1, all of the hypothetical evolutionary paths are potentially possible, which of them are probable? From a statistical standpoint we can assume the least probable is potential pathway 2 i.e., the development of high orders of molecular complexity leading to the intelligent interactivity of living systems. As has been noted (Carr \& Rees, 1979) with even a minor deviation in the value of the fine structure constant, electrons do not form stable orbitals around protons and a contingency like pathway 1 results.

As previously mentioned, the recent work by Susskind \& Maldacena that demonstrates the equivalence of maximally extended black holes in asymptotically anti-de Sitter space (which produces wormholes) with quantum entanglement in conformal field theory (AdS/CFT Correspondence theorem), suggests that entanglement is simply one manifestation of the Planckian wormhole network, such that other correlations (quantum correlations occur even in separable states (Auccaise et al., 2011) albeit weaker in strength or shared information content) 
also arise from the Planckian wormhole communication channels. We generalize this theorem to model multiply entangled systems, which would have a relatively higher Planckian wormhole density. One question that is being proposed here is if this Planckian wormhole network of higher order molecules could interact through temporal nonlocality (Figure 4), so as to affect previous (and future) interactions of the constituent subunits of molecules, influencing them to adopt high conformational orders of structural complexity, much like advanced potentials of the Wheeler-Feynman Absorber theory (Cramer, 1980).

Compare Figure 4 with Figure 1 and note that the vectors indicating the relative degree of probability (signified by the magnitude of the arrows) among the potential pathways are altered from the interaction of the Planckian wormhole network such that the highest probable potential path in Figure 1 becomes the lowest in Figure 4, and likewise the lowest in Figure 1 becomes the highest in Figure 4.

It is the potential temporal and spatial nonlocal interaction of physical systems, through the Planckian wormhole spacetime network that may produce an impetus, or veritable "force", that drives systems into increasingly higher orders of structural complexity and organizational synergy. The mechanism is driven by temporal nonlocal, retrocausal interaction. Systems with high orders of complexity contain many more potential states than lower orders of complexity. Moreover, the synergistic organizational arrangements of such systems may produce or involve greater degrees of strong correlation among the subunits of the system, so as to engender unity of the system.

\section{Result and Summary}

We have described a ubiquitous, unified connectivity and information system formed by the quantum wormhole network at the Planck scale of spacetime. The information encoding and processing functions of this basal and ubiquitous network merits the appellation spacememory (the unified spacememory network - the USN model). Evolution and developmental processes of physical systems, from non-organic to biological matter, are shaped by the integrative and ordering influences of this Planckian wormhole network, from cosmogenesis to the universe being aware of itself, i.e. consciousness.

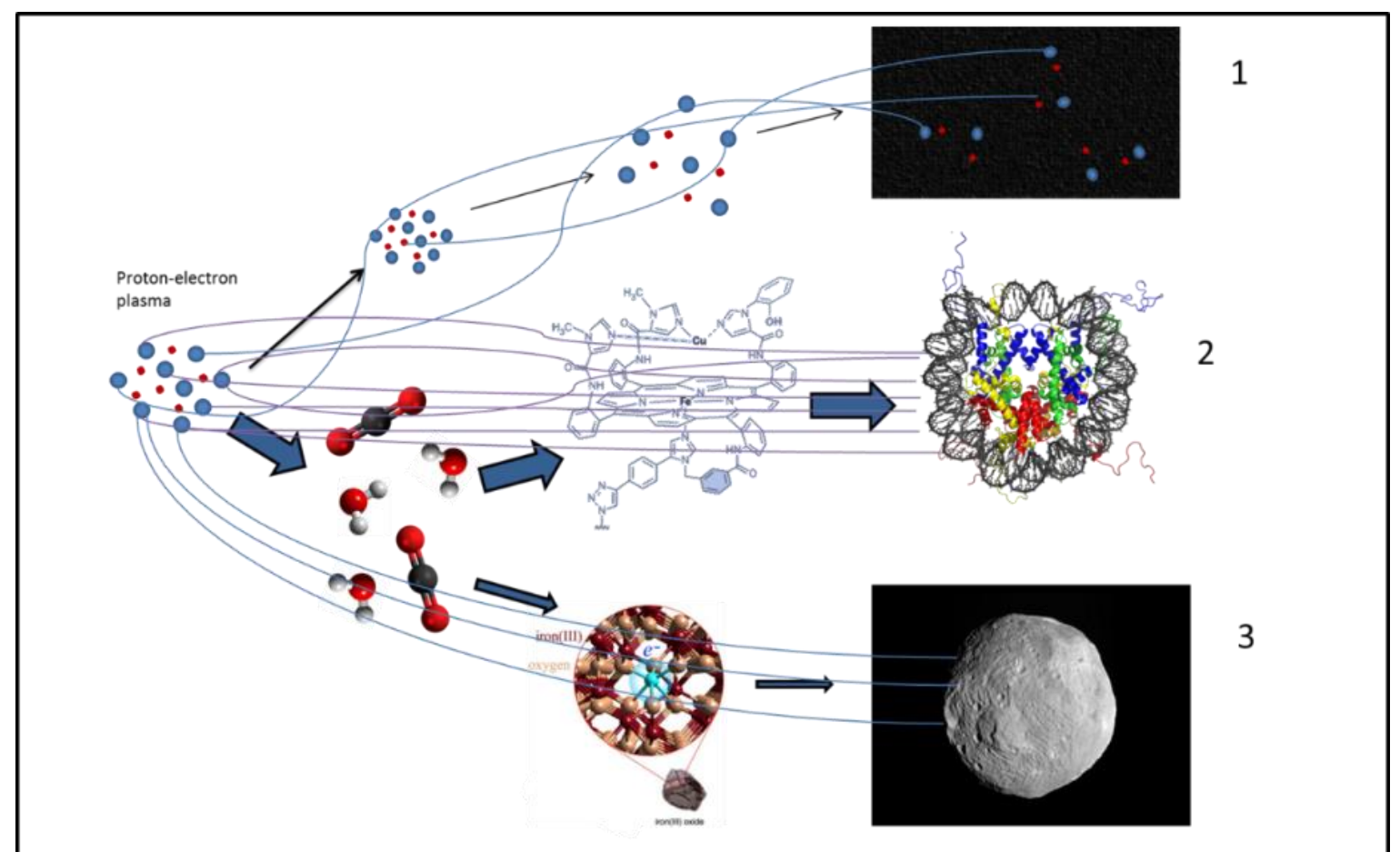

Figure 4. Postulated effect of nonlocal interactions (EPR correlations) of the Planckian wormhole information network on the development and evolution of atomic and molecular structures in the universe. The high density of Planckian wormhole connections integral to complex and highly ordered molecules (pathway 2) produce a stronger interaction across the temporal dimension, as well as intramolecularly. This influences the interactivity of atoms such that there is a higher impetus to form complex associations - a negentropic effect. 
To briefly reiterate and summarize the primary postulates of the USN model:

I: The constants of nature are not arbitrarily or randomly generated at the onset of the Big Bang2. They are defined by specific relationships and ratios of the holographic and quantum information structure of the Universe (such as the general holographic information relationship demonstrated in equations 3,5 , and 6).

- There is a certain conceptual framework within which this postulate is formulated, which has a couple of notable assumptions. The Universe did not emerge from nothing, nor from an indescribable point of singularity. It may have been generated as one of a multitude within a continuous multiverse landscape. As such, there may have been a pre-existing information structure, which is the product of holographic information relationships and ratios.

II: Following that the Planck-scale architecture of spacetime is comprised of polarizable electromagnetic quanta, which may have the capacity to encode information as Planck bits, or Planck voxels as we have defined them (equation 1 ), and the information encoding capacity of spacetime has non-zero hysteresis.

-With the specific values of the strength of forces and interactions emerging from the fundamental spacetime architecture at the onset of the Big Bang, and the memory encoding function of spacetime quanta, there is a self-ordering and self-organizational characteristic to physical systems, engendering an "initial" capacity for the formation of higher orders of complexity and organizational synergy.

III: Planck voxels are wormholes (equations 11 and 12), forming a filamentous Planckian network connecting all spacetime coordinates and quanta - mediating quasi-instantaneous (EPR, or nonlocal) correlations.

- The Planck-length-sized wormholes provide communication paths between spacetime coordinates, and quanta (such as baryons) in a quasi-instantaneous manner.

- This is a 'binding' principle, correlating subsystems across the Universe such that the information content of events are intercommunicated nonlocally.

- The communication between manifold subsystems or reference frames, information encoding (memory), and responsiveness (evolution or adaptability) naturally engender an awareness and sentience inherent to the spacememory network.

The living organism is comprised of a complex system of biomolecules intercommunicating in an intricate network of information and energy exchange. These highly complex biomolecules are in turn comprised of components that have evolved and developed under the influence of a larger, more fundamental intercommunication system, the Unified Spacememory Network (USN). At each scale there are sentient dynamics creating order and directionality of interaction towards higher levels of synergetic organization, and as a result, greater levels of consciousness. From the basal level of consciousness of the unified spacememory network, emerges higher orders of consciousness of the living organism. Until at the level of complexity exhibited by many animals, and undoubtedly human beings, there emerges selfawareness. The Planckian wormhole network connects spatial, temporal, and parallel domains, and mediates intercommunication, integration, and responsiveness of seemingly disparate systems across scales, engendering systems intelligence, and the biophilic and purposeful directionality of cosmological evolution and development from natural principles alone.
${ }^{2}$ Note that the current Standard Model utilizes 20 free parameters (depending whether neutrino parameters are considered), which are eISSN 1303-5150 not explained by causal mechanisms but instead are derived experimentally.

www.neuroquantology.com 
References

Attwater J, Wochner A, Pinheiro V, Coulson A, and Hollinger P. Ice as a protocellular medium for RNA replication. Nature Communications 2010; 1:76.

Auccaise R, Maziero J, Celeri L, Soares-Pinto O, and De Azevedo E. Experimentally witnessing the quantumness of correlations. Physical Review Letters 2011; 107(7):070501.

Baez J, Vicary J. Wormholes and entanglement. Classical and Quantum Gravity 2014; 31:11.

Bandyopadhyay A. Multi-level memory-switching properties of a single brain microtubule. Applied Physics Letters 2013; 123701: 102-114.

Barrow J and Tipler F. The anthropic cosmological principle. Oxford University Press. 1986.

Bekenstein J. Black Holes and Entropy. Physical Review D 1973; 7: 2333-2346.

Christian Borghesi, Julien Moukhtar, Matthieu Labousse, Antonin Eddi, Emannuel Fort, and Yves Couder. Interaction of two walkers: Wave-mediated energy and force. Physical Review E 2014. 90: 063017.

Braakman R and Smith E. The compositional and evolutionary logic of metabolism. Physical Biology 2013; 10:62.

Brosius J, Dull T, Noller H. Complete nucleotide sequence of a 23S ribosomal RNA gene from Escherichia coli. Proceedings of the National Academy of Sciences 1980; 77(1): 201-204.

Brosius J, Palmer M, Kennedy P, and Noller H. Complete nucleotide sequence of a $16 \mathrm{~S}$ ribosomal RNA gene from Escherichia coli. Proceedings of the National Academy of Sciences 1978; 75(11): 4801-4805.

Bush J. Pilot-wave hydrodynamics. Annual Review of Fluid Mechanics 2015; 47: 269-292.

Cantara W, Crain P, Rozenski J, Mccloskey J, Harris K, Zhang X, and Agris P. The RNA modification database, RNAMDB: 2011 update. Nucleic Acids Research 2011; 39(1): D195D201.

Carr B and Rees M. The anthropic principle and the structure of the physical world. Nature 1979; 278: 605-612.

Cramer JG. Generalized absorber theory and the EinsteinPodolsky-Rosen paradox. Physical Review D 1980; 22(2): 362-376.

Dawkins R. The Selfish Gene. Oxford University Press. 1989.

Del Giudice E, Spinetti PR, and Tedeschi A. Water dynamics at the root of metamorphosis in living organisms. Water 2010; 2 : 566-586.

Dennet D. Consciousness Explained. Little, Brown and Co. 1991.

Dworkin J, Deamer D, Sandford S, and Allamandola L. Selfassembling amphiphilic molecules: synthesis in stimulated interstellar/ precometary ices. Proceedings of the National Academy of Sciences 2000; 98(3): 815-819.

Freeman WJ and Vitiello G. Nonlinear brain dynamics as macroscopic manifestation of underlying many-body field dynamics. Physics of Life Reviews 2006; 3: 93-118.

Frohlich H. Long-range coherence and energy storage in biological systems. Internation Journal of Quantum Chemistry 1968; 2: 641-649

Fuller B and Applewhite E. Explorations in the geometry of thinking: Synergetics. Macmillan Publishing Co Inc. 1982.
Fuxreiter M, Mexei M, Simon I, and Osman R. Interfacial water as a "hydration fingerprint" in the noncognate complex of BamHI. Biophysical Journal 2005; 89: 903-911.

Garvalov BK, Zuber B, Bouchet-Marquis C, Kudryashev M, Gruska M, Beck M, Leis A, Frischknecht F, Bradke F, Baumeister W, Dubochet J, and Cyrklaff M. Luminal particles within cellular microtubules. Journal of Cell Biology 2006; 174(6): 759-765.

Hall M, Deckert DA and Wiseman J. Quantum phenomena modeled by interactions between many classical worlds. Physical Review X 2014; 4:041013.

Hameroff S. How quantum biology can rescue conscious free will. Frontiers in Integrative Neuroscience 2012; 6: 93.

Haramein N. Quantum gravity and the holographic mass. Physical Review and Research International 2013; 3:270 292.

Haramein $\mathrm{N}$ and Val Baker AKF. The Electron and the Holographic Mass. To be presented at the 4th Annual International Conference on Physics, Athens Greece, 2016; and available as online at https://www.researchgate.net/publication/302588440 The Electron and the Holographic Mass. Date Accessed: July 9th, 2016.

Hawking S. Particle creation by black holes. Communications in mathematical physics 1975; 43(3): 199-220.

Hayashi $\mathrm{T}$ and Stuchebrukhov A. Electron Tunneling in respiratory complex I. Proceedings of the National Academy of Sciences 2010; 107:19157-19162.

Hildner R, Brinks D, Nieder J, Cogdell R, and Hulst NF. Quantum coherent transfer over varying pathways in single lightharvesting complexes. Science 2013; 340: 1448-1451.

Hooft G. The holographic principle. Basics and Highlights in Fundamental Physics 2001; 37: 72-100 Hoyle F. The Intelligent Universe. London. Michael Joseph Ltd, 1983.

Lee KC, Sprague MR, Sussman BJ, Nunn J, Langford NK, Jin XM, Champion T, Michelberger P, Reim KF, England D, Jaksch D, Walmsley IA. Entangling macroscopic diamonds at room temperature. Science 2011; 334: 1253-1256.

Lemos J, Lobo F, Oliveira SQ. Morris-Thorne wormholes with a cosmological constant. Physical Review D 2003; 68: 6-15.

Maldacena J and Susskind L. Cool horizons for entangled black holes. Progress in Physics 2013; 61: 781-811.

Mavromatos NE, Mershin A, Nanopoulos DV. QED-cavity model of microtubules implies dissipationless energy transfer and biological quantum teleportation. International Journal of Modern Physics B 2002; 16: 3623.

Miranda G, Matos T, Garcia AN. Kerr-like Phantom Wormhole. General Relativity and Gravitation 2013. 46:1613.

Mohr P, Taylor B, Newell D. CODATA recommended values of the fundamental physical constants: 2010. Journal of Physical and Chemical Reference Data 2012; 41:043109.

Monnard PA and Ziock H. Eutectic phase in water-ice: a selfassembled environment conducive to metal-catalyzed non-enzymatic RNA polymerization. Chemistry and Biodiversity 2008; 5(8): 1521-1539

Nation P, Jahansson J, Blencowe $\mathrm{M}$, and Nori F. Stimulating uncertainty: amplifying the quantum vacuum with superconducting circuits. Review of Modern Physics 2012; 81(1):1. 
Haramein et al., The unified spacememory network

Ollivier H and Zurek W. Quantum discord: a measure of the quantumness of correlations. Physical Review Letters 2002; 88(1): 017901.

Panitcharyangkoon G, Hayes D, Fransted K, Caram J, Harel E, Wen J, and Engel G. Long-lived quantum coherence in photosynthetic complexes at physiological temperature. Proceedings of the National Academy of Sciences 2010; 107: 12766-12770.

Penrose R, Hameroff S, Stapp H, and Chopra D. Consciousness and the Universe: Quantum Physics, Evolution, Brain and Mind. Cosmology Science 2011.

Pohl R, Gilman R, Miller G, Pachucki K. Muonic hydrogen and the proton radius puzzle. Annual Review of Nuclear and Particle Science 2013; 63: 175-204.

Raamsdonk MV. Building up spacetime with quantum entanglement. General Relativity and Gravitation 2010; 42(10): 2323-2329

Reiper E, Anders J, and Vedral V. Quantum entanglement between electron clouds of nucleic acids in DNA. Physics 2011. arXiv: http://arxiv.org/pdf/1006.4053v2.pdf. Accessed date: July 5, 2016.

Sahu S, Ghosh B, Aswani K, Hirata K, Fujita D, and Bandyopadhyay A. Atomic water channel controlling remarkable properties of a single brain microtubule: correlating single protein to its supramolecular assembly. Biosensors and Bioelectronics 2013; 47:141-148.

Sheldrake R. Scientific papers on telepathy, the sense of being stared at, and morphic resonance. http://www.sheldrake.org/research. Accessed date: July 5, 2016.

Smolin L. Did the Universe evolve. Classical Quantum Gravity 1992; 9: 173-191.

Smolin L. Scientific alternatives to the anthropic principle. Physics 2004. arXiv: http://arxiv.org/pdf/hepth/0407213v3.pdf. Accessed Date: July 5, 2016.

Susskind

Thar R and Kuhl M. Propagation of electromagnetic radiation in mitochondria? Journal of Theoretical Biology 2004; 230(2): 261-270.

Tononi G. Consciousness as integrated information: a provisional manifesto. Biol Bull 2008; 215: 216-242.

Vidal C. Computational and biological analogies for understanding fine-tuned parameters in physics. Foundation of Science 2010; 15(4): 375-393.

Wagner A. Arrival of the fittest. Penguin Group: NY, New York City, 2014.

Wang H and Kais S. Quantum entanglement and electron correlation in molecular systems. Physics 2007. arXiv: http://arxiv.org/pdf/quant-ph/0701081v2.pdf. Date Accessed: July 5, 2016.

Weinberg S. The cosmological constant problem. Reviews of Modern Physics 1989; 61(1): 1-23.

Yang Y, Qin Y, Ding Q, Bakhtina M, Wang L, Tsai MD, and Zhong D. Ultrafast water dynamiccs at the interface of the polymerase-DNA binding complex. Biochemistry 2014; 53: 5405-5416.

Yves Couder and Emmanuel Fort. Single-particle Diffraction and Interference at a Macroscopic Scale. Physical Review Letters 2006. 97: 154101.

Zhang L, Yang Y, Kao YT, Wang L, and Zhong D. Protein hydration dynamics and molecular mechanism of coupled water - protein fluctuations. Journal of American Chemical Society 2009; 131: 10677-10691. 